\title{
GENERAL RELATIVISTIC SIMULATIONS OF ACCRETION INDUCED COLLAPSE OF NEUTRON STARS TO BLACK HOLES
}

\author{
Bruno Giacomazzo ${ }^{1}$ and Rosalba Perna ${ }^{2}$ \\ ${ }^{1}$ JILA, University of Colorado and National Institute of Standards and Technology, Boulder, CO 80309, USA \\ 2 JILA and Department of Astrophysical and Planetary Sciences, University of Colorado, Boulder, CO 80309, USA \\ Received 2012 July 12; accepted 2012 September 1; published 2012 September 20
}

\begin{abstract}
Neutron stars (NSs) in the astrophysical universe are often surrounded by accretion disks. Accretion of matter onto an NS may increase its mass above the maximum value allowed by its equation of state, inducing its collapse to a black hole (BH). Here we study this process for the first time, in three-dimensions, and in full general relativity. By considering three initial NS configurations, each with and without a surrounding disk (of mass $\sim 7 \% M_{\mathrm{NS}}$ ), we investigate the effect of the accretion disk on the dynamics of the collapse and its imprint on both the gravitational wave $(\mathrm{GW})$ and electromagnetic (EM) signals that can be emitted by these sources. We show in particular that, even if the GW signal is similar for the accretion induced collapse (AIC) and the collapse of an NS in vacuum (and detectable only for Galactic sources), the EM counterpart could allow us to discriminate between these two types of events. In fact, our simulations show that, while the collapse of an NS in vacuum leaves no appreciable baryonic matter outside the event horizon, an AIC is followed by a phase of rapid accretion of the surviving disk onto the newly formed BH. The post-collapse accretion rates, on the order of $\sim 10^{-2} M_{\odot} \mathrm{s}^{-1}$, make these events tantalizing candidates as engines of short gamma-ray bursts.
\end{abstract}

Key words: accretion, accretion disks - gamma-ray burst: general - gravitational waves - methods: numerical stars: neutron

Online-only material: color figures

\section{INTRODUCTION}

In the astrophysical universe, neutron stars (NSs) are often surrounded by an accretion disk. Several observations of X-ray binaries have indicated the presence of NSs in binary systems in which the NS accretes from the less-compact companion (e.g., Bildsten et al. 1997). Furthermore, isolated NSs are likely to be surrounded by a fallback disk remnant of the supernova explosion (e.g., Chevalier 1989), while numerical simulations have shown that the accretion induced collapse (AIC) of a white dwarf may lead to an NS surrounded by a disk with mass up to $\sim 0.8 M_{\odot}$ (Abdikamalov et al. 2010).

Even if the NS initially has a mass below the maximum limit, it can soon become unstable to gravitational collapse by accreting matter from the surrounding disk. Such AIC to a spinning black hole $(\mathrm{BH})$ could be a powerful source of gravitational waves (GWs), especially if the accreting matter excites non-axisymmetric modes in the NS before collapse. To date, no studies of such sources have been reported in full general relativity. On the other hand, the collapse of NSs (in vacuum) to $\mathrm{BHs}$ has been the subject of several investigations, including the accurate extraction of the GW signals emitted by these sources (Stark \& Piran 1985; Shibata et al. 2000; Shibata 2003; Baiotti et al. 2005a, 2005b, 2007; Baiotti \& Rezzolla 2006; Giacomazzo et al. 2011b). All these simulations have shown that the collapse is essentially axisymmetric and that no torus is left behind after the creation of a spinning BH. Because of this, the GW signals emitted by these sources will be rather weak and detectable only by advanced LIGO and Virgo if the sources were located in our Galaxy. Third generation detectors, such as the planned Einstein Telescope, could instead detect some of these sources up to a distance of $\sim 1 \mathrm{Mpc}$ (Giacomazzo et al. 2011b).

The AIC of an NS to a BH has also been suggested to be behind the central engine of short gamma-ray bursts (GRBs; e.g., see MacFadyen et al. 2005 and Dermer \& Atoyan 2006).
The idea is that an NS in a low-mass binary system could accrete enough mass from the companion star to produce a spinning $\mathrm{BH}$ surrounded by an accretion disk massive enough to power a relativistic jet and hence a short GRB.

Here we present the first numerical simulations describing the AIC of an NS to BH via fully three-dimensional general relativistic simulations. We follow the collapse of three initial NS configurations, each with and without an accreting torus, and study the impact of the accreting material on the dynamics of the collapse and its imprint on the GW signal, as well as the post-collapse evolution of the disk. Given that this is the first of such studies, we consider non-magnetized NSs. We also note that, while we do not expect magnetic fields to have a strong impact during the collapse (because, even when considering highly magnetized NSs, the ratio of magnetic to gas pressure is much lower than 1), they can have an important role after BH formation since they may extract energy from the system and power relativistic jets (Rezzolla et al. 2011).

Our Letter is organized as follows: In Section 2 we describe the initial NS/torus configurations, and the numerical setup used to study them. The dynamics of the collapse is reported in Section 3, while the GW signal emitted during the AIC event is described in Section 4, followed by a discussion (Section 5) of possible electromagnetic (EM) counterparts. We finally summarize and conclude in Section 6.

Throughout this Letter we use a spacelike signature of $(-,+,+,+)$ and a system of units in which $c=G=M_{\odot}=1$ (unless specified otherwise).

\section{INITIAL DATA AND NUMERICAL SETUP}

The initial data are built using the codes RNS (Stergioulas \& Friedman 1995; Nozawa et al. 1998; Stergioulas 2003) and TORERO (Corvino 2009). More specifically, the uniformly rotating NS models are built using the numerical code RNS 
Table 1

Properties of the Initial Models

\begin{tabular}{|c|c|c|c|c|c|c|c|c|c|c|c|c|}
\hline Model & $\begin{array}{c}\rho_{c} \\
\left(10^{15} \mathrm{~g} \mathrm{~cm}^{-3}\right)\end{array}$ & $r_{p} / r_{e}$ & $\begin{array}{c}M_{b} \\
\left(M_{\odot}\right)\end{array}$ & $\begin{array}{c}M \\
\left(M_{\odot}\right)\end{array}$ & $\begin{array}{c}R_{e} \\
(\mathrm{~km})\end{array}$ & $J / M^{2}$ & $C$ & $l_{0}$ & $\delta$ & $\begin{array}{c}R_{\text {in }} \\
(\mathrm{km})\end{array}$ & $\begin{array}{l}R_{\text {out }} \\
(\mathrm{km})\end{array}$ & $\begin{array}{l}M_{\text {torus }} \\
\left(M_{\odot}\right)\end{array}$ \\
\hline $1 \mathrm{a}$ & 1.91 & 0.85 & 1.90 & 1.73 & 12.2 & 0.36 & 0.21 & $\ldots$ & $\ldots$ & $\ldots$ & $\ldots$ & $\ldots$ \\
\hline $1 b$ & 1.91 & 0.85 & 1.90 & 1.73 & 12.2 & 0.36 & 0.21 & 3.63 & -0.60 & 13.6 & 31.6 & 0.141 \\
\hline $2 \mathrm{a}$ & 1.85 & 0.75 & 1.98 & 1.80 & 13.2 & 0.47 & 0.20 & $\ldots$ & $\ldots$ & $\ldots$ & $\ldots$ & $\ldots$ \\
\hline $2 b$ & 1.85 & 0.75 & 1.98 & 1.80 & 13.2 & 0.47 & 0.20 & 3.61 & -0.74 & 14.7 & 32.5 & 0.143 \\
\hline $3 a$ & 1.85 & 0.65 & 2.05 & 1.86 & 14.4 & 0.55 & 0.19 & $\ldots$ & $\ldots$ & $\ldots$ & $\ldots$ & $\ldots$ \\
\hline $3 b$ & 1.85 & 0.65 & 2.05 & 1.86 & 14.4 & 0.55 & 0.19 & 3.60 & -0.80 & 15.5 & 33.4 & 0.145 \\
\hline
\end{tabular}

Notes. The columns refer respectively to the name of the model, the central value $\rho_{c}$ of the rest-mass density of the NS, the ratio $r_{p} / r_{e}$ of the polar to equatorial radius of the NS, the baryonic $\left(M_{b}\right)$ and gravitational $(M)$ masses of the NS, the proper equatorial radius $R_{e}$, the ratio $J / M^{2}$ of the total angular momentum and gravitational mass, and the compactness $C \equiv M / R_{e}$. The disk is characterized by the parameters $l_{0}$ and $\delta$ (see the text for description), its inner $\left(R_{\mathrm{in}}\right)$ and outer $\left(R_{\text {out }}\right)$ radius, and its baryonic mass $M_{\text {torus }}$.

with a polytropic equation of state (EOS), $p=K \rho^{\Gamma}$, where $p$ and $\rho$ are pressure and rest-mass density, respectively, while $K=100$ and $\Gamma=2$ are the polytropic constant and the polytropic exponent, respectively. All the initial models are chosen to be on the unstable branch (Takami et al. 2011) in order to be able to compare the GW signal between a collapsing NS with and without a torus. In the former case we assume that the torus has already accreted enough mass on the NS to make it unstable, and we follow its dynamics after that point. The NS collapse is triggered by reducing its pressure by $0.1 \%$ at the beginning of the simulations.

When we add an accretion disk to the rotating NS, we use the code TORERO, which can compute stationary solutions of axisymmetric disks around spinning BHs by solving the equations described in Daigne \& Font (2004) and Corvino (2009). In these equations the self-gravity of the disk is neglected and hence they are valid only when the mass of the disk is sufficiently small compared to the mass of the central compact object. We note that, even if the torus is built assuming the spacetime of a spinning $\mathrm{BH}$, the violation of the Hamiltonian constraint introduced by having the torus orbiting around a spinning NS is not large enough to affect the dynamics of the system. This is due to the fact that the tori considered here have masses that are a small fraction of the mass of the NS. We have further verified that this violation decreases with increasing resolution and that it is negligible compared to the violations introduced during the evolution (i.e., the infinity norm of the Hamiltonian constraint is similar during the collapse between the models with and without a torus). Furthermore, the initial violation produced by adding the torus produces a spurious burst of radiation that leaves the grid well before the beginning of the collapse and hence it does not contaminate the GW signal computed from these models. The tori are built by giving as input parameters to TORERO the gravitational mass $M$ and the ratio of angular momentum to gravitational mass, $J / M^{2}$, of the NS, and two parameters $l_{0}$ and $\delta$ which define, respectively, the initial value of the constant angular momentum in the disk and the depth level reached by the disk inside the potential well. A negative value of $\delta$ represents a stable solution, while a positive value would give a torus overflowing its Roche lobe.

Table 1 lists the relevant properties of the NSs and tori studied here. We have chosen tori with masses $\sim 7 \%$ of the mass of the NS in order to have a non-negligible effect on the dynamics.

The evolution of these NS/torus configurations is computed using the fully general relativistic magnetohydrodynamic code Whisky (Baiotti et al. 2005a; Giacomazzo \& Rezzolla 2007; Giacomazzo et al. 2011a), which adopts a flux-conservative formulation of the equations and high-resolution shock-capturing schemes. All the results presented here have been obtained by means of the Piecewise Parabolic Method, while the Harten-Lax-van-Leer-Einfeldt approximate Riemann solver has been used to compute the fluxes (Baiotti et al. 2005a; Giacomazzo \& Rezzolla 2007). All the simulations were performed using an ideal-fluid EOS with $\Gamma=2$. Magnetic field effects were not included in these simulations and they will be the subject of future work. The evolution of the spacetime was obtained using the Ccatie code, a three-dimensional finitedifferencing code providing the solution of a conformal traceless formulation of the Einstein equations (Pollney et al. 2007). Both the Ccatie and Whisky codes benefit of the use of the Einstein Toolkit (Löffler et al. 2012).

The simulations were run using the vertex-centered fixed mesh-refinement approach provided by the Carpet driver (Schnetter et al. 2004). We have used seven refinement levels with the finest resolution being $0.1 M_{\odot} \approx 0.148 \mathrm{~km}$ and the coarsest resolution being $6.8 M_{\odot} \approx 10.04 \mathrm{~km}$. The finest grid has a radius of $10 M_{\odot} \approx 14.77 \mathrm{~km}$ (which is sufficient to completely cover the NS), whereas the coarsest grid extends to $998.4 M_{\odot} \approx 1474 \mathrm{~km}$. For all the simulations reported here we have also used a reflection-symmetry condition across the $z=0$ plane and a $\pi$-symmetry condition across the $x=0$ plane. ${ }^{3}$

\section{DYNAMICS}

Figure 1 shows the evolution of the rest-mass density $\rho$ on the equatorial $x y$ plane for models $3 \mathrm{a}$ (left panels) and $3 \mathrm{~b}$ (right panels). We remind the reader that the only difference between the initial data of these two models is the fact that we added a torus in the case of model $3 \mathrm{~b}$. The top panels show the initial data, the center ones the time of collapse to $\mathrm{BH}$, and the bottom panels the end of the simulations. While the dynamics is essentially axisymmetric ${ }^{4}$ as it was already seen in the case of the collapse of NSs without a disk (Baiotti et al. 2007; Giacomazzo et al. 2011b), the main difference is that the torus resists the gravitational collapse and continues to orbit and accrete onto the spinning $\mathrm{BH}$ (bottom-right panel). Moreover, since the accreting torus triggers oscillations in the NS density, the collapse is slightly delayed in the case in which the NS is surrounded by a disk. This happens because the NS oscillations prevent the central density from growing exponentially from the

\footnotetext{
3 Stated differently, we evolve only the region $\{x \geqslant 0, z \geqslant 0\}$ and apply a $180^{\circ}$-rotational-symmetry boundary condition across the plane at $x=0$.

4 In order to assess the impact of the use of $\pi$-symmetry on our results, we also performed some simulations without using it, and we verified that the dynamics is not affected and that the evolution remains axisymmetric.
} 

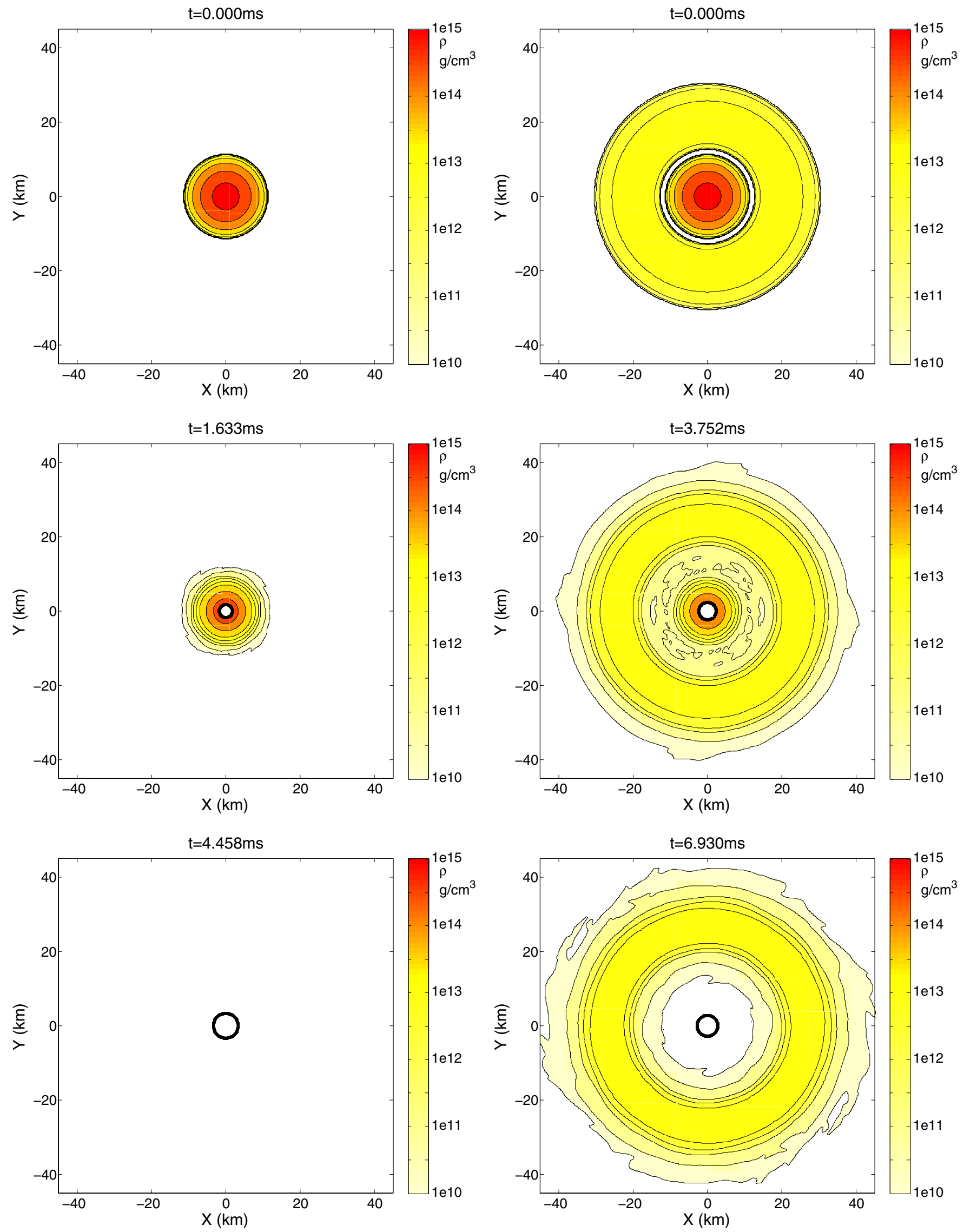

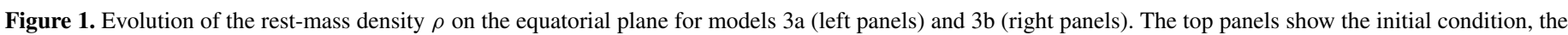

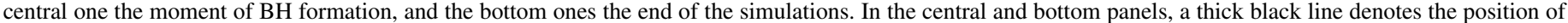
the apparent horizon of the $\mathrm{BH}$.

(A color version of this figure is available in the online journal.) 

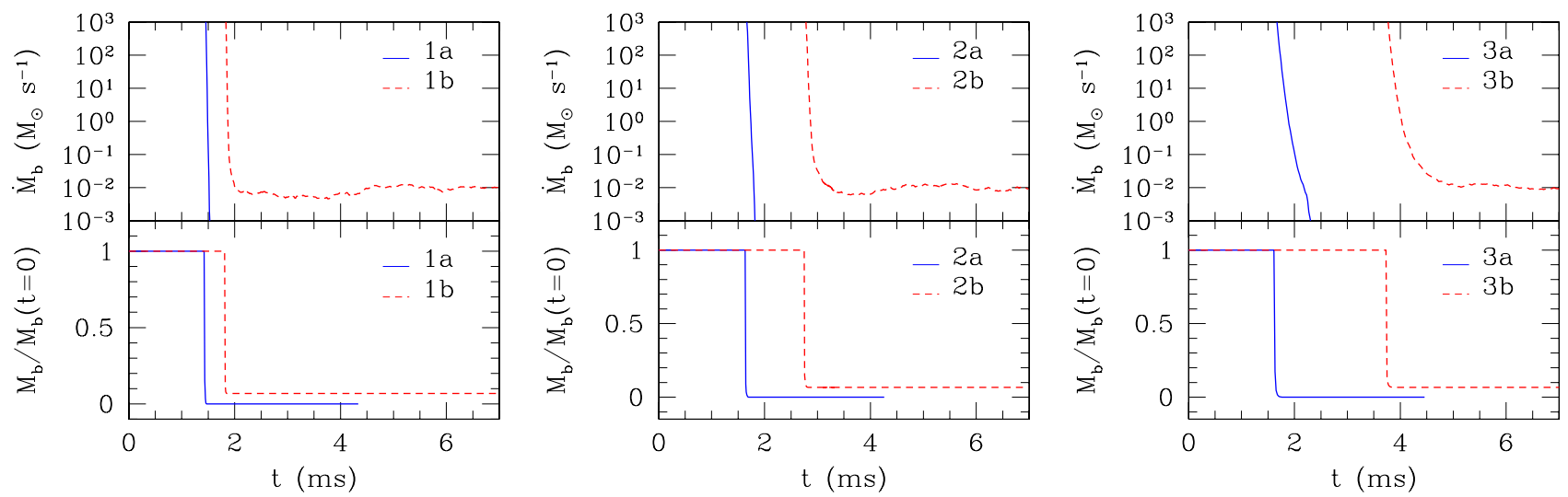

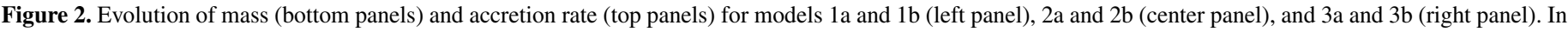
all panels, the blue solid line refers to the case without a torus (1a, $2 a, 3 a)$ and the red dashed line to the case with a torus (1b, 2b, 3b).

(A color version of this figure is available in the online journal.)

very beginning, since each NS oscillation produces an expansion and hence a small decrease of its central density. This qualitative dynamics is similar also in the other cases we studied (models $1 \mathrm{a} / 1 \mathrm{~b}$ and $2 \mathrm{a} / 2 \mathrm{~b}$ ). In the bottom panels of Figure 2 we show the evolution of the total baryonic mass $M_{b}$, normalized to its initial value. All the cases which included a torus at the beginning (red dashed lines) still preserve $\sim 7 \%$ of the initial mass outside the $\mathrm{BH}$ at the end of the simulations. On the other hand, all the models without a torus (blue solid lines) do not leave any mass outside the $\mathrm{BH}$, as it was already seen in previous simulations of the collapse of an NS to a $\mathrm{BH}$ in vacuum ${ }^{5}$ (Baiotti et al. 2007; Giacomazzo et al. 2011b). Another important quantity is the accretion rate $\dot{M}_{\mathrm{b}}$ after the formation of the $\mathrm{BH}$ (top panels of Figure 2). We compute this as the integral of the flux of matter that crosses the apparent horizon. In all the models that left a torus outside the $\mathrm{BH}$, its early-time value is found to be $\sim 10^{-2} M_{\odot} \mathrm{s}^{-1}$.

We note that in our simulations (as well as in previous generalrelativistic simulations of NS-NS mergers), the accretion is triggered both by numerical viscosity and by the spacetime dynamics. In order to assess the contamination from the numerical component (i.e., the numerical viscosity), we performed simulations at different resolutions, and found that the accretion rate remained at the same level. Hence we can safely conclude that the contribution from numerical dissipation is negligible in our results. However note that, in astrophysical disks, turbulent viscosity, likely generated by the magneto-rotational instability (Balbus \& Hawley 1991), causes angular momentum transport and hence accretion. For an $\alpha$-viscosity parameter $\sim 0.1$ (Shakura \& Sunyaev 1973), we found that the viscous timescale in our torus is $t_{0} \sim 0.1 \mathrm{~s}$, which would yield an even larger post-collapse accretion rate $\dot{M} \sim M_{\text {torus }} / t_{0} \sim 1 M_{\odot} \mathrm{s}^{-1}$. Since this estimate is highly dependent on the disk properties and its viscosity, we note that our computed post-collapse $\dot{M}_{\mathrm{b}}$ should be rather considered as a lower limit to what can be achieved by the system following an AIC event.

\section{GRAVITATIONAL WAVES}

Figure 3 displays the GW signal emitted by the AIC of an $\mathrm{NS}$ to a $\mathrm{BH}$ in our six models, and in particular the dominant $l=2, m=0$ mode computed using the Weyl scalar $\Psi_{4}$ (Baker

\footnotetext{
5 Note that the presence of magnetic fields alters these conclusions. Simulations of magnetized, differentially rotating NSs (Duez et al. 2006a, 2006b) show that, following the NS collapse, the BH is surrounded by a hot torus.
}

et al. 2002; Löffler et al. 2012). The blue solid lines refer to the cases without a torus, while the red dashed lines show the signal emitted when the torus is present. Since the collapse is essentially axisymmetric, $l=2, m=0$ is the dominant mode, and the signal is given by an exponential increase followed by the ring-down of the $\mathrm{BH}$. No significant difference can be seen in the signal between the models with and without a torus, except for the fact that the GWs emitted by the models with a torus show some small oscillations before the collapse. These are related to the NS oscillations triggered by the accretion of the disk, as previously mentioned in Section 3. Similarly to the case of the collapse of an NS in vacuum, the GW emission would be detectable by advanced LIGO/Virgo only if the sources were located in our Galaxy. On the other hand, with the Einstein Telescope, the signal could be detected for sources at distances of up to $\sim 1 \mathrm{Mpc}$.

\section{ELECTROMAGNETIC EMISSION}

As described in Section 3, the presence of a rapidly accreting torus left around the newly born $\mathrm{BH}$ is a distinctive feature of the AIC, differentiating it from the direct collapse of an NS into a $\mathrm{BH}$, which leaves no mass for the $\mathrm{BH}$ to accrete from.

The accretion rates that our AIC events predict (cf. Figure 2), on the order of $\sim 10^{-2} M_{\odot} \mathrm{s}^{-1}$, are within an order of magnitude of those obtained in the merger of two NSs (e.g., Rezzolla et al. 2010). For a typical mass-to-energy conversion efficiency $\sim 0.1$, these AICs have luminosities sufficiently high to constitute enticing candidates for short GRBs. In fact, assuming an energyto- $\gamma$-ray conversion efficiency $\sim$ several tens of percent (as expected for short GRBs, e.g., Zhang et al. 2007), our simulated AIC events would have peak luminosities in $\gamma$-rays on the order of $\sim 10^{51} \mathrm{erg} \mathrm{s}^{-1}$, compatible with at least a fraction of short GRBs. Clearly, the predicted accretion rates are dependent on the torus mass, and a more comprehensive exploration will be performed in future work.

Short GRBs from the AIC of an NS in a binary are envisioned to have the observer located out of the binary plane (MacFadyen et al. 2005), and a jet launched along the NS rotation axis-either as the result of $v \bar{v}$ annihilation (e.g., Aloy et al. 2005) or of magnetohydrodynamic effects (e.g., Drenkhahn \& Spruit 2002); furthermore, they are expected to be more often found in early-type or globular cluster environments (Dermer \& Atoyan 2006). A detailed computation of their expected cosmological rates, and a comparison with the observed population of short GRBs, is reserved for future work. In particular, we will 

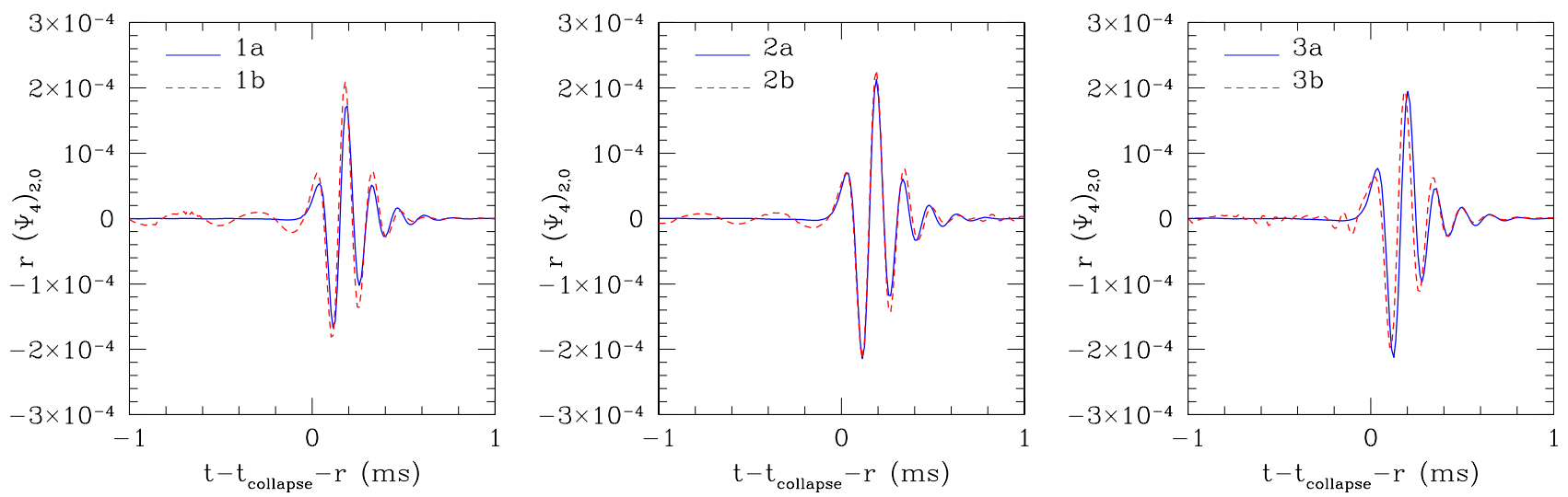

Figure 3. Gravitational wave signal $r\left(\Psi_{4}\right)_{(l=2, m=0)}$ for models $1 \mathrm{a}$ and $1 \mathrm{~b}$ (left panel), 2a and $2 \mathrm{~b}$ (center panel), and $3 \mathrm{a}$ and $3 \mathrm{~b}$ (right panel). All the signals are extracted at $r=100 M_{\odot} \approx 148 \mathrm{~km}$. In all panels, the blue solid line refers to the case without a torus (1a, 2a, 3a) and the red dashed line to the case with a torus (1b, 2b, 3b). The time is shifted by the time of collapse $t_{\text {collapse }}$ (defined as the moment at which an apparent horizon is formed).

(A color version of this figure is available in the online journal.)

examine the dependence of the AIC accretion rates on the disk mass, and hence assess the likelihood of their occurence in $\mathrm{X}$-ray binaries (where disk masses are smaller than the ones considered here) and fallback disks around isolated NSs (e.g., Perna et al. 2000 and references therein). Finally, within the connection short GRBs/AIC events, we further note that the observed X-ray flares (Margutti et al. 2011) and extended emissions (Norris \& Bonnell 2006), lasting up to several hundreds of seconds, cannot be directly explained as the result of activity from the central engine (which in an AIC is much shorter than the flare duration), unless the disk undergoes some instability (e.g., Perna et al. 2006; Proga \& Zhang 2006).

\section{CONCLUSIONS}

We have performed the first three-dimensional, fully general relativistic simulation of the AIC of an NS to a $\mathrm{BH}$; we have examined the GW signal expected from such events, and computed the accretion rates onto the newly formed $\mathrm{BH}$. We find that the main component $(l=2, m=0$ mode) of the GW signal is very similar to the one from the collapse of an NS into a BH in vacuum. However, and most importantly, while the direct collapse of a non-magnetized NS onto a BH leaves no mass behind (hence making unlikely the presence of any EM detectable counterpart), an AIC is followed by rapid accretion of the disk onto the newly formed BH. Our computed accretion rates, comparable to those obtained in simulations of NS-NS mergers, make AIC events potential engines for short GRBs. NSs in X-ray binaries (e.g., Bildsten et al. 1997), isolated NSs accreting from fallback disks (Chevalier 1989), and NSs produced by the AIC of a white dwarf (Abdikamalov et al. 2010) are astrophysical scenarios which might host the AIC of an NS onto a $\mathrm{BH}$.

We thank Giovanni Corvino for providing us with the code TORERO and Roland Haas for assistance in computing the accretion rates. We also thank Eleonora Troja, Cole Miller, and the anonymous referee for carefully reading the manuscript and for their useful comments. Numerical simulations were performed on the cluster RANGER at the Texas Advanced Computing Center (TACC) at The University of Texas at Austin through XSEDE grant No. TG-PHY110027. B.G. and R.P. acknowledge support from NSF grant No. AST 1009396.

\section{REFERENCES}

Abdikamalov, E. B., Ott, C. D., Rezzolla, L., et al. 2010, Phys. Rev. D, 81, 044012

Aloy, M. A., Janka, H.-T., \& Muller, E. 2005, A\&A, 436, 273

Baiotti, L., Hawke, I., Montero, P. J., et al. 2005a, Phys. Rev. D, 71, 024035

Baiotti, L., Hawke, I., \& Rezzolla, L. 2007, Class. Quantum Grav., 24, 187

Baiotti, L., Hawke, I., Rezzolla, L., \& Schnetter, E. 2005b, Phys. Rev. Lett., 94, 131101

Baiotti, L., \& Rezzolla, L. 2006, Phys. Rev. Lett., 97, 141101

Baker, J., Campanelli, M., \& Lousto, C. O. 2002, Phys. Rev. D, 65, 044001

Balbus, S. A., \& Hawley, J. F. 1991, ApJ, 376, 214

Bildsten, L., Chakrabarty, D., Chiu, J., et al. 1997, ApJS, 113, 367

Chevalier, R. A. 1989, ApJ, 346, 847

Corvino, G. 2009, PhD thesis, Università degli studi di Parma

Daigne, F., \& Font, J. A. 2004, MNRAS, 349, 841

Dermer, C. D., \& Atoyan, A. 2006, ApJ, 643, L13

Drenkhahn, G., \& Spruit, H. C. 2002, A\&A, 391, 1141

Duez, M. D., Liu, Y. T., Shapiro, S. L., Shibata, M., \& Stephens, B. C. 2006a, Phys. Rev. Lett., 96, 031101

Duez, M. D., Liu, Y. T., Shapiro, S. L., Shibata, M., \& Stephens, B. C. 2006b, Phys. Rev. D, 73, 104015

Giacomazzo, B., \& Rezzolla, L. 2007, Class. Quantum Grav., 24, 235

Giacomazzo, B., Rezzolla, L., \& Baiotti, L. 2011a, Phys. Rev. D, 83, 044014

Giacomazzo, B., Rezzolla, L., \& Stergioulas, N. 2011b, Phys. Rev. D, 84, 024022

Löffler, F., Faber, J., Bentivegna, E., et al. 2012, Class. Quantum Grav., 29, 115001

MacFadyen, A. I., Ramirez-Ruiz, E., \& Zhang, W. 2005, arXiv: astro-ph/0510192

Margutti, R., Chincarini, G., Granot, J., et al. 2011, MNRAS, 417, 2144

Norris, J. P., \& Bonnell, J. T. 2006, ApJ, 643, 266

Nozawa, T., Stergioulas, N., Gourgoulhon, E., \& Eriguchi, Y. 1998, A\&AS, 132, 431

Perna, R., Armitage, P. J., \& Zhang, B. 2006, ApJ, 636, L29

Perna, R., Hernquist, L., \& Narayan, R. 2000, ApJ, 541, 344

Pollney, D., Reisswig, C., Rezzolla, L., et al. 2007, Phys. Rev. D, 76, 124002

Proga, D., \& Zhang, B. 2006, MNRAS, 370, L61

Rezzolla, L., Baiotti, L., Giacomazzo, B., Link, D., \& Font, J. A. 2010, Class. Quantum Grav., 27, 114105

Rezzolla, L., Giacomazzo, B., Baiotti, L., et al. 2011, ApJ, 732, L6

Schnetter, E., Hawley, S. H., \& Hawke, I. 2004, Class. Quantum Grav., 21, 1465

Shakura, N. I., \& Sunyaev, R. A. 1973, A\&A, 24, 337

Shibata, M. 2003, Phys. Rev. D, 67, 024033

Shibata, M., Baumgarte, T. W., \& Shapiro, S. L. 2000, Phys. Rev. D, 61, 044012

Stark, R. F., \& Piran, T. 1985, Phys. Rev. Lett., 55, 891

Stergioulas, N. 2003, Living Rev. Rel., 6, 3, http://www.livingreviews.org/ lrr_2003-3

Stergioulas, N., \& Friedman, J. L. 1995, ApJ, 444, 306

Takami, K., Rezzolla, L., \& Yoshida, S. 2011, MNRAS, 416, L1

Zhang, B., Liang, E., Page, K. L., et al. 2007, ApJ, 655, 989 